Wei Song*, Yanju Yang and Jin Li

\title{
Crystal structure of (E)-3-chloro-2-(((4- nitrophenyl)imino)methyl)phenol, $\mathrm{C}_{13} \mathrm{H}_{9} \mathrm{ClN}_{2} \mathrm{O}_{3}$
}

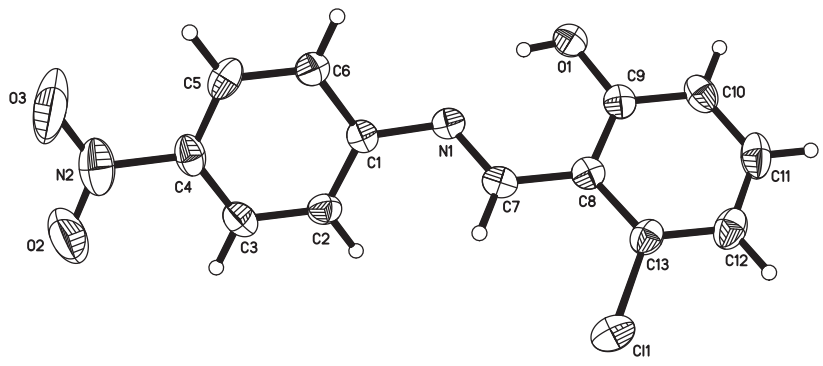

https://doi.org/10.1515/ncrs-2019-0476

Received July 7, 2019; accepted August 30, 2019; available online September 11, 2019

\section{Abstract}

$\mathrm{C}_{13} \mathrm{H}_{9} \mathrm{ClN}_{2} \mathrm{O}_{3}$, orthorhombic, $P 22_{1} 2_{1} 2_{1}$ (no. 19), $a=7.401$ (3) $\AA$, $b=12.005(5) \AA, \quad c=13.808(5) \AA, \quad V=1226.8(8) \AA^{3}, \quad Z=4$, $R_{\mathrm{gt}}(F)=0.0673, w R_{\text {ref }}\left(F^{2}\right)=0.1473, T=293(2) \mathrm{K}$.

\section{CCDC no.: 1950104}

The asymmetric unit of the molecular structure is shown in the figure. Table 1 contains crystallographic data and Table 2 contains the list of the atoms including atomic coordinates and displacement parameters.

\section{Source of material}

4-Nitroaniline (1 mmol, $138.1 \mathrm{mg})$ and 2-chloro-6hydroxybenzaldehyde ( $1 \mathrm{mmol}, 156.6 \mathrm{mg}$ ) were added to the solution of ethanol $(20 \mathrm{~mL})$ containing a few drops of glacial acetic acid, then the mixture was refluxed for $6 \mathrm{~h}$. The title compound was obtained after the evaporation of the solvent. The solid product was dissolved in acetone and the solution was evaporated slowly at room temperature. Yellow needle crystals suitable for X-ray diffraction determination were obtained after $4 \mathrm{~d}$.

\section{Experimental details}

All $\mathrm{H}$ atoms were positioned geometrically and refined as riding atoms, with $\mathrm{C}-\mathrm{H}=0.93 \AA$ and $\mathrm{O}-\mathrm{H}=0.82 \AA$. The $U_{\text {iso }}$

*Corresponding author: Wei Song, School of Biological and Chemical Engineering, Nanyang Institute of Technology, 473004 Nanyang, Henan, P.R. China, e-mail: songweinyang@163.com. https://orcid.org/0000-0003-3644-5880

Yanju Yang and Jin Li: School of Biological and Chemical Engineering, Nanyang Institute of Technology, 473004 Nanyang, Henan, P.R. China

Ә Open Access. ( 2019 Wei Song et al., published by De Gruyter. (cc) BY License.
Table 1: Data collection and handling.

\begin{tabular}{ll}
\hline Crystal: & Yellow needle \\
Size: & $0.22 \times 0.16 \times 0.12 \mathrm{~mm}$ \\
Wavelength: & Mo $K \alpha$ radiation $(0.71073 \AA)$ \\
$\mu:$ & $0.32 \mathrm{~mm}^{-1}$ \\
Diffractometer, scan mode: & Bruker APEX-II, $\varphi$ and $\omega$ \\
$\theta_{\text {max }}$, completeness: & $25.0^{\circ}, 99 \%$ \\
$N(h k l)_{\text {measured }}, N(h k l)_{\text {unique }}, R_{\text {int }}:$ & $6049,2133,0.081$ \\
Criterion for $I_{\text {obs }}, N\left(h k l l_{\text {gt }}:\right.$ & $I_{\text {obs }}>2 \sigma\left(I_{\text {obs }}\right), 1408$ \\
$N(\text { param })_{\text {refined }}:$ & 173 \\
Programs: & Bruker [1], SHELX [2] \\
\hline
\end{tabular}

Table 2: Fractional atomic coordinates and isotropic or equivalent isotropic displacement parameters $\left(\AA^{2}\right)$.

\begin{tabular}{lrrrr}
\hline Atom & $\boldsymbol{x}$ & $\boldsymbol{y}$ & $\boldsymbol{z}$ & $\boldsymbol{U}_{\text {iso }}^{*} / \boldsymbol{U}_{\text {eq }}$ \\
\hline C1 & $0.6012(8)$ & $0.3132(5)$ & $0.6511(4)$ & $0.0294(14)$ \\
C2 & $0.5397(8)$ & $0.2335(5)$ & $0.7155(4)$ & $0.0377(16)$ \\
H2 & 0.490050 & 0.167737 & 0.691938 & $0.045^{*}$ \\
C3 & $0.5508(8)$ & $0.2500(6)$ & $0.8133(4)$ & $0.0414(16)$ \\
H3 & 0.508172 & 0.196186 & 0.856093 & $0.050^{*}$ \\
C4 & $0.6261(8)$ & $0.3473(6)$ & $0.8477(4)$ & $0.0419(17)$ \\
C5 & $0.6832(8)$ & $0.4293(6)$ & $0.7854(4)$ & $0.0419(17)$ \\
H5 & 0.730911 & 0.495311 & 0.809611 & $0.050^{*}$ \\
C6 & $0.6695(7)$ & $0.4133(5)$ & $0.6875(4)$ & $0.0362(15)$ \\
H6 & 0.705672 & 0.469268 & 0.645102 & $0.043^{*}$ \\
C7 & $0.6296(7)$ & $0.2042(5)$ & $0.5125(4)$ & $0.0327(15)$ \\
H7 & 0.660422 & 0.145641 & 0.553450 & $0.039^{*}$ \\
C8 & $0.6236(7)$ & $0.1844(5)$ & $0.4096(4)$ & $0.0313(14)$ \\
C9 & $0.5690(8)$ & $0.2683(5)$ & $0.3442(4)$ & $0.0348(15)$ \\
C10 & $0.5667(8)$ & $0.2495(6)$ & $0.2464(4)$ & $0.0426(17)$ \\
H10 & 0.533403 & 0.306334 & 0.204329 & $0.051^{*}$ \\
C11 & $0.6136(9)$ & $0.1466(7)$ & $0.2103(5)$ & $0.0519(19)$ \\
H11 & 0.608477 & 0.134019 & 0.143918 & $0.062^{*}$ \\
C12 & $0.6683(8)$ & $0.0618(6)$ & $0.2715(5)$ & $0.0465(17)$ \\
H12 & 0.701216 & -0.007314 & 0.246636 & $0.056^{*}$ \\
C13 & $0.6730(8)$ & $0.0812(5)$ & $0.3689(4)$ & $0.0385(16)$ \\
C11 & $0.7461(3)$ & $-0.02596(13)$ & $0.44412(12)$ & $0.0605(6)$ \\
N1 & $0.5938(6)$ & $0.3000(4)$ & $0.5499(3)$ & $0.0322(11)$ \\
N2 & $0.6464(9)$ & $0.3631(8)$ & $0.9518(5)$ & $0.0684(19)$ \\
01 & $0.5216(6)$ & $0.3709(3)$ & $0.3766(3)$ & $0.0454(12)$ \\
H1 & 0.537398 & 0.374190 & 0.435314 & $0.068^{*}$ \\
O2 & $0.6006(11)$ & $0.2873(6)$ & $1.0059(4)$ & $0.106(3)$ \\
03 & $0.7025(11)$ & $0.4513(8)$ & $0.9804(4)$ & $0.132(3)$ \\
\hline & & & &
\end{tabular}

values were refined with $U_{\text {iso }}(\mathrm{H})=1.2 U_{\text {eq }}(\mathrm{C})$ and $1.5 U_{\text {eq }}(0)$. The absolute structure was established by refinement of the Flack parameter (0.14(8) from 422 selected quotients) using Parsons' method [3].

This work is licensed under the Creative Commons Attribution 4.0 Public 


\section{Comment}

For decades Schiff-bases and their derivative chemistry have gained increasingly more and more attention due to their simple synthetic and wide applications in the fields of coordination chemistry, biochemistry, and analytical chemistry [4-9]. In order to expand the research of Schiff bases, the title compound was synthesized and its crystal structure determined.

The asymmetric unit of the title compound consists of one formula unit ( $c f$. the figure). The molecule displays an $E$ configuration. In the crystal structure of the title compound, the $\mathrm{C}=\mathrm{N}$ double bond length is $1.289(7) \AA(\mathrm{C} 7=\mathrm{N} 1)$, exhibiting the double bond character. The title compound is a non-planar molecule. The dihedral angle between the two aromatic ring systems is $42.46(1)^{\circ}$. The nitro group is nearly co-planar with its aryl ring making a dihedral angle of $3.2^{\circ}$. In the crystal structure, molecules are linked into one-dimensional chains by weak $\mathrm{C}-\mathrm{H} \cdot \mathrm{O}$ O hydrogen bonds. One intramolecular $\mathrm{O}-\mathrm{H} \cdots \mathrm{N}$ hydrogen bond exists, which stabilizes the conformation of the title molecule (see the figure). Geometric parameters are similar to that of the parent structure [10].

Acknowledgements: This work was financially supported by Nanyang Institute of Technology.

\section{References}

1. Bruker. APEX2, SAINT and SADABS. Bruker AXS Inc., Madison, WI, USA (2008).
2. Sheldrick, G. M.: A short history of SHELX. Acta Crystallogr. A64 (2008) 112-122.

3. Parsons, S.; Flack, H. D.; Wagner, T.: Use of intensity quotients and differences in absolute structure refinement. Acta Crystallogr. B69 (2013) 249-259.

4. Zong, H.; Hong, Y.; Liu, W.; Wu, J.: Crystal structure of $(E)$ 2-(1-((2-aminophenyl)imino)ethyl)-4,6-dichlorophenol, $\mathrm{C}_{14} \mathrm{H}_{12} \mathrm{Cl}_{2} \mathrm{~N}_{2}$ O. Z. Kristallogr. NCS 234 (2019) 595-596.

5. Chang, F. F.; Zhang, L.; Zhao, P. C.; Huang, W.: Transmetalation for flexible pendant-armed Schiff-base macrocyclic complexes influenced by halide effects. Inorg. Chem. 57 (2018) 1438-1448.

6. Shokohi-Pour, Z.; Chiniforoshan, H.; Sabzalian, M. R.; Esmaeili, S. A.; Momtazi-borojeni, A. A.: Cobalt(II) complex with novel unsymmetrical tetradentate Schiff base (ON) ligand: in vitro cytotoxicity studies of complex, interaction with DNA/protein, molecular docking studies, and antibacterial activity. J. Biomol. Struct. Dyn. 36 (2018) 532-549.

7. Asadizadeh, S.; Amirnasr, M.; Tirani, F. F.; Mansouri, A.; Schenk, K.: DNA-BSA interaction, cytotoxicity and molecular docking of mononuclear zinc complexes with reductively cleaved $\mathrm{N}_{2} \mathrm{~S}_{2}$ Schiff base ligands. Inorg. Chim. Acta 483 (2018) 310-320.

8. Shanty, A. A.; Philip, J. E.; Sneha, E. J.; Kurup, M. R. P.; Balachandran, S.; Mohanan, P. V.: Synthesis, characterization and biological studies of Schiff bases derived from heterocyclic moiety. Bioorg. Chem. 70 (2017) 67-73.

9. Luo, W. L.; Shi, T.; Liu, S. F.; Zuo, W. W.; Li, Z. B.: Well-designed unsymmetrical salphen-Al complexes: synthesis, characterization, and ring-opening polymerization catalysis. Organometallics 36 (2017) 1736-1742.

10. Burgess, J.; Fawcett, J.; Russell, D. R.; Gilani, S. R.; Palma, V.: Four $\mathrm{N}$-(2-hydroxybenzylidene)aniline derivatives. Acta Crystallogr. C55 (1999) 1707-1710. 